\title{
Nature of L2 Grammatical Proficiency: A Multi-Dimensional Approach
}

\author{
Ahmad Reza Lotfi \\ Islamic Azad University- Khorasgan Branch \\ P.O.BOX: 81595-158
}

Tel: 98-913-317-8603 E-mail: arlotfi@yahoo.com

Behrouz Nouri Samani

Islamic Azad University- Khorasgan Branch

P.O.BOX: 81595-158

Tel: 98-311-260-1115Ｅ-mail: Behrouznourisamani@yahoo.com

\begin{abstract}
This is a study with descriptive hypothesis-testing design investigating the true nature of L2 grammatical proficiency via focusing on its component parts. It examines the performance of 60 female EFL learners between 19 and 30 years of age at 2 proficiency levels using 2 types of computerized Grammaticality Judgement tasks in different orders (i.e. Explicit Written Task and Implicit Written Task) for each participant at a 1 week interval for 2 weeks per each task to determine the extent to which L2 learners' performances are affected by a few components of grammatical proficiency chosen and manipulated out of many others that exist (i.e. Implicit Knowledge, and Explicit knowledge) as well as language proficiency while focusing on subjacency. Participants rendered GJ tasks on a 2-point interval. Results from analyzing the elicited measurement data indicate that the performance scores of the two proficiency groups in the two Grammaticality Judgement tasks did not differ significantly.
\end{abstract}

Keywords: Explicit Written GJ task, Grammaticality Judgement task, Implicit Written GJ task, L2 grammatical proficiency, subjacency

\section{Introduction}

A growing body of research has indicated the importance of syntax ever since the influence of Chomsky's ideas on linguistics. Within syntax, an important syntactic operation exists (i.e. merger) which allows words, phrases and clauses to form sentence structures. In fact, it is this operation which allows the formation of grammatical sentences in all languages; that is, words combine to form phrases, phrases combine to form clauses, and clauses combine to form complex sentences. The rationale behind the operation merger is that all languages, English included, have a potentially infinite number of sentences which can be in the form of interrogative sentences, declarative sentences, and the like.

Such complex syntactic structures are the result of the successive merging of pairs of categories to form larger phrases. In fact, such structures serve as projections of overt constituents such as words, phrases, and clauses. Moreover, such syntactic structures consist of covert, null, and empty constituents. For example, one of the important aspects of syntactic structures is the empty category which is of immense significance in the formation of WH-questions. One sample of such empty categories is the trace left behind as the result of different movement types. Traces are of significance in the processing of different sentences, and tree diagrams, which are binary-branching diagrams, represent different syntactic structures, and consist of three positions (i.e. specifier, head and complement) (Radford, Atkinson, Britain, Clahsen \& Spencer, 2009).

In fact, the syntactic structures of sentences are independent of their related meanings, hence the ability to study such syntactic structures independently of their associated semantic contents. However, the sentential ordering of syntactic structures, according to Chomsky, only permit specific orderings, hence the ungrammaticality of some sentences in GJ tasks. A variety of grammar models, which are derivations of UG, try to explain the rationale behind such specific orderings and explore the computational properties of humans responsible for the syntactic processing of sentences in general. Hence, Universal Grammar is an appropriate choice in this regard because it is of great complexity and power in syntax.

Syntactic computation, which is a major property of human language faculty, is structure dependent. That is, humans have to consider structural hierarchies if they intend to perform syntactic operations. In addition, humans' language faculty can compute and build such binding relations as c-command and indexation that are parts of the resources that constitute their language faculty (Isac \& Reiss, 2008). Syntax is a universal combinatory system of 
humans' linguistic competence, which is an integral part of language faculty, and constitutes the basis for all human language instantiations (Ramchand, 2008).

\subsection{WH-Questions}

Interrogatives are one type of complex syntactic structures and are of two types (i.e. closed and open). Whereas the former shows subject-auxiliary inversion, the latter shows fronting of the nonsubject interrogative phrase that involves a WH-word with the ability to trigger subject-auxiliary inversion, and, as a result, the occurrence of WH-movement. The criterion for classifying all complex syntactic structures, which are in the form of questions, is the answer types that follow them; that is, questions are either yes/no questions, alternative questions because of the presence of or, or WH-questions. WH-questions express a variable in their proposition, and it is the substitution of this variable with a value that gives rise to answers to such WH-questions. In addition, such WH-questions can be in the form of multiple WH-questions (Collins, 2006). In fact, interrogatives are multiclausal sentences that are syntactically related to each other via subordination because their clauses show unequal syntactic status. Noun (complement), adjective (relative) and adverb clauses are three types of subordinate clauses in English (Huddleston \& Pullum, 2006).

\subsection{Movement}

An important pertinent issue within WH-questions is movement. Movement is a process in generative grammar that was formulated from the early 1970s. In fact, movement is a syntactic operation by which constituents move and leave behind silent trace copies. Furthermore, movement operations "move as few constituents as possible the shortest distance possible" (Economy Principle) (Radford et al. 2009, p.301). In 2000s, traces, which came into existence as the result of movement, were reformulated in terms of copying according to which units in one position were duplicated in another (Matthews, 2007).

Movements are of two types, that is A-movements and A-bar movements. In cases where an expression, such as an $\mathrm{NP}$, is moved to a position that can only be occupied by argument expressions, such as the case of passivization, A-movement occurs, whereas in cases where an expression, such as WH-phrases, is moved to a position that can be occupied by both arguments and adjuncts, A-bar movement occurs (Radford, 2004). Another type of syntactic movement is head-movement such as the cases where a V moves to I (Hawkins, 2001).

Movement can occur both overtly (i.e. before spell-out) and covertly (i.e. after spell-out at LF). Different causes trigger different movement types such as feature checking, which triggers WH-movements. One of the major characteristics of movements is the resultant chains that are formed between the moved WH-phrases (head of the chain) and the traces left behind by such movements (foot of the chain). Moreover, movements are constrained by a UG principle termed the subjacency principle (Radford, 1997). The syntactic form of movement acts as a distinguishing factor among languages and, as a result, differs from language to language. Whereas in some languages like Japanese and Chinese, questions are formed without movement, in others, such as English, they require movement. Variable movements exist in other languages such as the case of Turkish (Cook, 1993).

\subsection{Subjacency}

Subjacency, which was formulated by Chomsky in the late 1970s, refers to the syntactic constraints that block the movement of a unit if that unit crosses more than one bounding node. WH-movement is constrained by subjacency, and refers to the movement of WH-forms to the beginning of a sentence or even a clause resulting in the formation of WH-traces. The extracted constituents make short-step moves because long moves give rise to ungrammaticality in constructions. In addition, the $\mathrm{Q}$ morpheme of complementizer requires WH-phrases to move into the clause-initial specifier CP-position.

\subsubsection{Subjacency in English and Persian}

Whereas IPs and NPs (DPs) are the bounding nodes in English, NPs and CPs are the bounding nodes in Persian. Hence, WH-movement must be, in English, to the nearest empty CP to avoid subjacency violations, whereas it must be, in Persian, to the nearest empty IP to avoid subjacency violation. Subjacency is an example of "subtle parameter variation" that extends far beyond L2 input and cannot be developed by L1 grammar. In fact, within the sphere of bounding nodes, parametric variation is seen across languages (White, 1989, p.111). In some languages where WH-forms are not preposed, such as Persian, WH-forms are called in-situ; however, in English such WH-in-situ questions are used as echo questions. In fact, feature strength triggers operator movements in English. In English, the WH-feature of probe is strong; hence, the noninterpretable feature of the probe attracts its interpretable counterpart on goal overtly, that is before spell-out, and the moved WH-form c-commands its trace (Radford, 1997).

Checking requirements trigger the movement of operator expressions. Checking checks and deletes the non-interpretable formal features, which have no semantic content, upon reaching LF because such features are the 
viruses of the system, unlike the interpretable formal features that are reserved and interpreted. In fact, WH-phrases either move to check (i.e. the operation Attract, or rather Enlightened Self-Interest) or to be checked (i.e. the operation Greed) (Lotfi, 2003).

\subsection{WH-Islands}

Many of the UG principles are parameterized because they function with varying degrees of effects in different languages. As for the principle of subjacency, it should be noted that, when it comes to the issue of movement, certain construction types are islands; that is, extraction is impossible out of them. Such syntactic islands fall into four categories, namely object noun complements, subject noun complements, complex NPs, and WH-islands. Accordingly, certain general constraints limit the freedom of the WH-movement rules such as the A-over-A condition, subject condition, the complex noun phrase constraint (CNPC), and WH-island constraint. All of these constraints can be reduced to the cases of subjacency (White, 1988).

\subsection{The Nature of Grammar}

Grammar is a property of individual minds the nature of which individuals are unaware of (Isac \& Reiss, 2008). In fact, the grammar of a language refers to all the rules and principles of that language and is observationally adequate, that is it provides a systematic description of sentence formation in different languages (Haegeman, 1991).

Every language is made up of linguistic ingredients, and such ingredients are arranged based on a set of rules which are called grammar. One part of grammar that deals with the sentence structures is called syntax. As such, the major concern of syntax is word order, and those linguists who work within generativism have taken into account syntactic universals as the best piece of evidence for Universal Grammar. Such universals have even been of constructive contribution in SLA research because they apply to all language types (Magni, Scalise \& Bisseto, 2009).

In fact, grammar is a single and complex unit that generates syntactic structures and assigns representations to such structures at two levels, namely phonological form (PF) and logical form (LF). Different opinions have existed about the grammar structure since the start of generative linguistics in the 1950s; however, all such different opinions have agreed on one fact, that is grammar is abstract, and is not located in space or time. A speaker's grammar is his knowledge object because such a grammar contains information about his language. In addition, such a grammar is represented in the speakers' minds. Grammars serve in the speaker/hearers' mental processes and mediate the production and perception of speech. Moreover, the study of grammar is highly effective in understanding the nature of linguistic ability of humans because when people speak a language, they follow the formal rules of it, such as rules of syntax (George, 1989).

Understanding the nature of grammar enables researchers to understand the true nature of language. Whereas descriptive grammar describes a speaker's basic linguistic knowledge, the rules that he already knows and represents the unconscious linguistic knowledge of speakers because of being a model of the mental grammar that every speaker has, prescriptive grammar legislates what a grammar should be (Fromkin, Rodman \& Hyams, 2003).

\subsection{Implicit and Explicit Knowledge}

L2 acquisition theories distinguish between two types of linguistic knowledge, that is implicit and explicit knowledge. Whereas some researchers (e.g., Dekeyser, 1998) adopt an interface position where explicit instruction leads to the development of both explicit and implicit knowledge, others (e.g., Doughty, 2003) adopt the position where explicit knowledge only leads to the development of 'explicit' knowledge.

Instruction, which is an attempt to intervene the interlanguage development process, is of two types, namely communication-focused instruction and form-focused instruction. Whereas the former directs learners' attention to meaning, the latter directs learners' attention to form. Implicit and Explicit instruction types exist within the form-focused instruction (FFI). Implicit form-focused instruction attracts learners' attention to target forms; it is delivered spontaneously; it is unobtrusive where meaning communication is minimally interrupted; it presents contextualized target forms; it does not use metalanguage; and, finally, it encourages target forms to be used freely.

On the other hand, explicit form-focused instruction directs (and does not attract) learners' attention to target forms; it is planned and predetermined; it is obtrusive where meaning communication is interrupted; it presents target forms in isolation; it does use metalanguage terminology; and, finally, encourages target forms to be practiced in a controlled way. Hence, the stance where explicit and implicit instruction types are distinguished is favored over the one where they are not distinguished.

In the explicit instruction type, learners focus on some rule during their learning process and develop metalinguistic awareness towards that rule. In fact, it is a deductive process where a rule is given to the learners. Furthermore, explicit instruction is directed towards intentional and explicit learning. In the implicit instruction type, on the other 
hand, learners are directed to infer rules without awareness, hence its being inductive in process. Implicit instruction is directed towards incidental learning.

Whether implicit and explicit knowledge types are separate or two poles on a continuum remains a controversial issue. However, most researchers (e.g., Ellis, 2005) have argued for the former case. Likewise, some other researchers (e.g., Krashen, 1981) have argued against the existence of interaction between these two knowledge sources, whereas others have argued for the existence of an interface position.

\subsection{Research Question}

Is change in the explicit-implicit dichotomy in the ways of stimuli presentations in Grammaticality Judgement tasks accompanied by a corresponding change in female EFL participants' performance while rendering WH-questions?

\subsection{Research Hypothesis}

Change in the explicit-implicit dichotomy in the ways of stimuli presentations in Grammaticality Judgement tasks will be significantly accompanied by a corresponding change in female EFL participants' performance while rendering WH-questions; in other words, those female EFL participants who will be explicitly involved in rendering WH-questions will be significantly more successful in rendering WH-questions than those female EFL participants who will be implicitly involved in rendering WH-questions.

\subsection{Statement of the Problem}

The specific problem under study in this research is to determine the extent to which High- and Low-Intermediate EFL learners can observe subjacency constraints while performing operator movements under different conditions controlled by the explicit-implicit dichotomy because language is a part of our mental structure and a cognitive system; as a result, different means of receiving linguistic input have different effects on our language processing such as the difference between receiving input via pictures (i.e. Implicit Written Task), visual sentences (i.e. Explicit Written Task) or otherwise. The reason is that different psychological processes are triggered by such different input types. Hence, the purpose of the study is the determination of the effects of explicit and implicit forms of GJ stimuli presentations on female EFL participants' performance in GJ tasks while rendering WH-questions in English. That is, the intention is to determine whether explicit and implicit forms of stimuli (WH-questions) presentations yield similar or dissimilar performance types in female EFL participants when such participants are tested on subjacency violations (WH-questions) in Grammaticality Judgement tasks, and, in case of the existence of dissimilar performance types, which form is of more positive effects on female EFL participants.

\subsection{Significance of the Study}

The importance of this problem lies in investigating the true nature of EFL learners' grammatical proficiency while studying subjacency (operator movements) using two different Grammaticality Judgement Tasks where some subcomponents of grammaticality proficiency are controlled such as the effect of implicit-explicit dichotomy on High- and Low-Intermediate EFL learners' performance in the way different stimuli are presented to them within Explicit and Implicit Grammaticality Judgement tasks. In fact, each approach (i.e. each of the two approaches adopted while analyzing the two types of GJ Tasks [Explicit and Implicit Written Tasks]) will examine grammatical proficiency from a different angle to see if different approaches trigger distinct levels of comprehension of English WH-questions on EFL female participants' part, a case which, if proved, could lead to a major shift in SLA researchers' view towards EFL learners' grammatical proficiency because grammatical proficiency would no longer be viewed as a unified solid whole, but a unit made of numerous component parts with each part being triggered by a distinct task type. The approach to solving the problem is to statistically analyze the within and between-group performance of the two proficiency levels across two WH-movement GJ tasks over two consecutive weeks.

The primary theoretical implication of the study will be research-bound largely because researchers will get familiar with the nature of GJ tasks further; that is, different researchers will be able to determine whether GJ tasks can be used for effectively measuring different components of participants' grammatical proficiency; moreover, researchers can determine the degree to which GJ tasks are successful in reliably measuring different component parts of L2 learners' grammatical proficiency. The linguistic implication of the study will be to gain a better understanding towards the true nature of L2 grammatical proficiency (i.e. whether grammatical proficiency is a unified whole unit as has always been claimed to be the case by different linguists and SLA researchers such as Lydia White, or a unit consisting of different component parts where each part contributes differently to the functioning of grammatical proficiency). The pedagogical implications will be finding out better ways of improving the grammatical proficiency of L2 learners via adopting new teaching methods that can target different grammatical proficiency component parts more effectively and lead to a better development and a higher proficiency level of L2 learners' grammatical proficiency. In addition, teachers can adopt more effective drills and exercises in the course of teaching L2 learners 
different principles of subjacency in their L2 because such teachers have got more familiar with the nature of L2 learners' grammatical proficiency. Language teachers can also change their stance towards the issue of language proficiency because, given the results of this study, they will get a better understanding towards the degree to which L2 learners' language proficiency level can be of significant effects in improving the performance of L2 learners in different tests.

In fact, the theoretical implications of the study can change our approach towards the current methods of teaching WH-questions at schools, universities, and like academic settings. Moreover, they can lead to the revision of the established theories of L2 learners' grammatical proficiency and the learning of WH-questions because subjacency is one aspect of how languages work, and facts about the existence of differences between the explicit and implicit modes of presenting different WH-questions, where subjacency has been violated, can lead to the development of new teaching methods where explicit and implicit (i.e. via pictures) ways of teaching can be distinguished in terms of their instructive features. The resultant formative assessment of such tasks can be used for curriculum evaluation, curriculum construction, modifying subsequent learning experiences, prescribing remedies for group and individual deficiencies, and addressing individual learners' needs.

Lastly, the findings of this study can assist test developers to find out whether they can use GJ tasks for reliably testing the nature of L2 learners' grammatical proficiency. In addition, such findings can encourage test developers to develop more reliable tests and design more meticulous tests for testing the overall nature of L2 grammatical proficiency.

\section{Literature Review}

Much research in subjacency (e.g., Schachter, 1988) has indicated that different L2 learners' access to the UG principle of subjacency and the proper observance of it in Grammaticality Judgement tasks can be either attributed to UG or the effect of L2 learners' L1, that is in cases where L2 learners' L1 resembles L2 (e.g. English) in terms of WH-movements, L2 learners' observance of subjacency could be either due to UG or their L1 (such as the case of Dutch native speakers learning English), whereas in cases where L2 learners' L1 does not resemble L2 in WH-movement, L2 learners' observance of subjacency could only be due to UG (such as the case of Korean native speakers learning English). In addition, such researchers have only tested their L2 learners' knowledge of subjacency under uniform and like conditions and have oftentimes concluded that subjacency is a part of the L2 learners' unconscious knowledge (or rather grammatical proficiency) although it may not be consistently obeyed. Other researchers (e.g., Bley-Vroman, Felix \& Loup, 1988) have only emphasized UG availability while rendering GJ tasks on subjacency, and have concluded that this availability of UG is, at times, only overridden by other factors in performance (White, 1990).

In another experiment, researchers (e.g., Schachter, 1989. 1990) have indicated that learners fail to recognize violations of UG principles, such as subjacency, unless these principles also operate in their L1; therefore, Korean and Chinese speakers cannot recognize subjacency violations in English, whereas Dutch speakers can (White, 1996). Research on the effect of proficiency in the observance of subjacency (e.g., White, Travis \& Maclachlan, 1992) has indicated that all the High-Intermediate adult Malagasy learners of English, and only half of the Low-Intermediate group has performed like a native English-speaking control group. In addition, results have proved that L1 is not the only source of the learners' UG-like knowledge, and UG principles remain available in adult L2 acquisition. This obtained result has, however, contradicted Bley-Vroman's belief that states that access to UG is only via the L1. Moreover, it has opposed the belief of those who claim no access to UG at all such as Clahsen and Muysken (1986) (White, 1996).

Research on L2 learners' role of implicit and explicit knowledge in rendering WH-questions on GJ tasks (e.g., Green \& Hecht, 1992) has indicated that tests show different kinds of sensitivity to the subjects' implicit and explicit knowledge. That is, in most cases, guessing on the students' part overestimates their implicit knowledge, and their facing difficulty in formulating a rule, based on which to decide between two competing forms, underestimates their explicit knowledge (Dekeyser, 2003).

There is one major caveat to these research types. Although some researchers (e.g., Schachter, 1988) have investigated the source of L2 learners' access to the subjacency principle in L2 to be either UG, L1 or both, and other researchers (e.g., Bley-Vroman, Felix \& Loup, 1988) have mostly concluded that subjacency is part of L2 learners' grammatical proficiency, few, if any, researchers have reported the actual nature of L2 grammatical proficiency. The few researchers who have attempted to relate UG-accessibility to maturity effects (e.g., Johnson \& Newport, 1991) have considered the matured and developed L2 grammatical proficiency to be a unified solid whole; no researcher has reported grammatical proficiency to be a unit made of numerous component parts with each part being triggered by a distinct task type. Thus, research to this time has measured only the subjacency performance of 
L2 learners on WH-movement GJ tasks focusing either on proficiency, source of L2 knowledge, the relationship between implicit-explicit knowledge, and performance with the assumption that grammatical proficiency is a unified solid whole and irrespective of their implications of their research findings on the nature of L2 grammatical proficiency.

Researchers (e.g., Cleeremans \& McClelland, 1991) have claimed that implicit learning is restricted when subjects are to establish relationships between distant elements in sentences. Moreover, subjects' ability in grammaticality has not provided hard evidence to prove that abstract issues are learned implicitly. Researchers have constantly argued that only simple knowledge can be implicitly learned and not more complex knowledge. Much research (e.g., Ellis, 2003) has indicated that a generative account of language makes a distinction between implicit and explicit knowledge. The former implies the manifestation of language knowledge in performance with no awareness; and the latter refers to that language knowledge of which speakers are aware and can verbalize. In fact, in rendering implicit grammatical judgements, subjects are at a loss when it comes to explaining how they can make a correct grammatical judgement. The widely-held belief is that whereas implicit knowledge is easy to have rapid access to, explicit knowledge is not, but proficiency is of effect in this regard; that is, with constant practice, access to explicit knowledge can be increased; however, some researchers (e.g., Hulstijn, 2002) have believed that practice only results in "false automatization", and cannot resemble implicit knowledge. Research on the relationship between explicit and implicit knowledge (e.g., Bialystok, 1992) has indicated that the nature of this relationship is different in L2 from L1; that is, in L1, children first acquire implicit knowledge of their L1, and, afterwards, they develop explicit knowledge out of L1 by analyzing it; hence, implicit knowledge forms the basis of explicit knowledge, whereas in L2, it is the other way round except for the cases of naturalistic exposure where implicit L2 knowledge serves as the pillar for explicit L2 knowledge development. However, in classrooms, L2 learners learn explicit knowledge.

\section{Method}

\subsection{Participants}

Sixty female EFL university students of the Islamic Azad University of Khorasgan (aged 19 to 30) participated in our study. Participants were randomly selected and divided into two proficiency groups (i.e. High-Intermediate and Low-Intermediate groups), as determined by the Grammar and Listening Modules of Oxford Placement Test (OPT) (2004). Participants had been permanent residents of Iran, and lived in Esfahan. Participants were treated according to the "Ethical Principles of Psychologists and Code of Conduct" (American Psychological Association, 2001).

\subsection{Materials}

Two two-choice Grammaticality Judgement tasks with the same number of WH-questions were used to tap female EFL participants' knowledge of forming WH-questions. The tasks examined the effects of two independent variables on the EFL participants' performances in WH-question GJ tasks; that is, the implicit-explicit dichotomy of the way stimuli were presented in two GJ tasks; and the L2 proficiency level of participants participating in the experiment.

The first GJ task, Explicit Writen Task, was a 30-question form-focused task based on which female EFL participants' explicit knowledge of constraints governing WH-movements in English was tested; that is, we instructed the participants to determine whether the written WH-questions served as proper questions for the explicitly written stimuli that preceded such WH-questions four seconds ago. Implicit Witten Task, on the other hand, was a 30-question meaning-focused task based on which female EFL participants' use of congruence strategy was tested; that is, we instructed the participants to determine whether the presented onscreen pictures were congruent with their respective WH-questions which would pop up on the screens four seconds after the pictures. We used AUTHORWARE software (2005), an SAT-based software (i.e. speed and accuracy tradeoff), to develop the two Grammaticality Judgement tasks.

The laptop used for collecting data from participants was of the brand DELL with processor traits as 'Intel(R) Core $^{\mathrm{TM}}$ 2Duo CPU, and T9300@2.50 GHz'; memory (RAM) capacity as $4.00 \mathrm{~GB}$; and system type as 32-bit operating system.

The tasks tested different cases of subjacency: (a) A-Over-A Condition where WH-elements are extracted out of an object noun complement; (b) Subject Condition where WH-elements are extracted out of a subject noun complement; (c) Complex Noun Phrase Constraint (CNPC) where WH-elements are extracted out of a complex NP; and (d) WH-Island Constraint where WH-elements are extracted out of a wh-clause. In all such subjacency cases more than one bounding node (NP, PP, S or Ś) has been crossed. 
The GJ tasks included both grammatical and ungrammatical WH-questions of equal complexity, vocabulary and structure. In addition, filler items were included in all the tasks. Participants were instructed in the two GJ tasks to select only one of the two choices for each question, namely either acceptable or not acceptable. The tasks investigated syntactic islands in English, that is those construction types that are islands with respect to movement.

\subsection{Design and Procedure}

The research strategy in his study was to manipulate the independent variables (i.e. Implicit Written Grammaticality Judgement task and Explicit Written Grammaticality Judgement task) to determine their effects on the dependent variable (i.e. female EFL participants' performance on the two WH-movement GJ tasks). We also used a repeated measures experimental research design in this study to test the hypothesis and the problem via administering all the two GJ tasks to all the participants over two weeks.

Testing occurred in two sessions for each participant 1 week apart. In each session, one of the two GJ tasks was used for each participant, and in another session, the other GJ task was used for the same participant. The presentation of the two GJ tasks was counterbalanced for the two groups. That is, in the first session, Explicit Written GJ task was used for the first group, whereas Implicit Written GJ task was used for the second group; in the second session, Implicit Written GJ task was used for the first group, whereas Explicit Written GJ task was used for the second group.

The control features used were the randomization in the selection of participants and counterbalancing that was implemented by not only changing the order of tasks administered to each participant, but also changing the order of question items in the same tasks used for different participants. To gather the required experimental data, we dedicated a coding sheet to each participant so that we would be able to record the scores of each participant.

Having received thorough verbal instructions on how to take the tasks, each participant would take her seat and use the laptop to take part in the experiment. On the Explicit Written task, participants, first, received a written stimulus in the form of a sentence for each item that was then followed by a related WH-question four seconds later to be judged for grammaticality. Each written stimulus and its respective WH-question would remain on the laptop screen until the participants answered it. Each item was a two-choice question, namely acceptable and not acceptable. We instructed the participants to click the left button if they found the WH-questions acceptable, and the right button if they found them not acceptable. On the Implicit Written task, the stimuli were in the form of pictures; that is, each question item consisted of a picture that would, first, appear on the screen and be then followed by a related WH-question to be judged for congruency.

Each participant took part in the computerized GJ tasks separately in a totally empty university class where no distractions existed at all. Further, an attempt was made to observe the same class lighting, day time for administering the tests, amount of words used in giving instructions, silence of the class, and other ambient conditions for each participant so that each participant would take the tests in nearly similar testing conditions.

\subsection{Statistical Procedures}

The actual steps carried out to conduct this study include the measurement device used that is the statistical test of two way repeated measures ANOVA. We used the two-way ANOVA for testing the hypothesis (where the effects of two independent variables [i.e. explicit and implicit ways of stimuli presentations in GJ tasks] on female EFL participants' performance [i.e. dependent variable] were tested).

Since we intended to compare participants' performance on more than one GJ task, we used the repeated measures ANOVA to be able to compare the overall performance of the High-Intermediate Group with the overall performance of the Low-Intermediate group. Each participant in both High- and Low-Intermediate groups did all the two GJ tasks. As a result, we had four sets of results to be compared for each group; that is, two sets of scores from the performance of High-Intermediate group on both Explicit and Implicit Written GJ tasks, and two sets of scores from the performance of Low-Intermediate group on both Explicit and Implicit Written GJ tasks.

Each group was analyzed separately via using repeated measures ANOVA in order to measure how a single group performed on multiple measures (i.e. the two GJ tasks), and to measure if the effects of those measures were independent. In addition, the overall performance of one group was statistically compared with the overall performance of the other group to see if proficiency was of any effects on the overall performance of the groups.

\section{Results}

Having entered the obtained data into the variable SPSS data view window with their descriptive labels, we obtained the indispensable general tendencies in the data and the overall spread of the scores via analyzing measures of central tendency and variability as shown in Table 1. 
The numerical values of mean indicate that HG-EXP-WRI did better than the other three independent variables because the mean value is weighted in its favor. The numerical values of standard deviation and variance give us exact ideas of the typical behavior of the two groups and show that HG-EXP-WRI has the least variability (i.e. more homogeneous in terms of performance on the Explicit Written GJ task) whereas LG-EXP-WRI has the most variability, and is, as a result, less homogeneous. Hence, HG-EXP-WRI shows the most degree of homogeneity whereas LG-EXP-WRI shows the least degree of homogeneity.

Moreover, the calculated standard deviation statistics portrayed in Table 1 shows how scores are dispersed around the means. Examining the standard deviation and means values in relation to one another, we found that LG-IMP-WRI and HG-EXP-WRI were more or less equally dispersed from the means, whereas HG-IMP-WRI and LG-EXP-WRI were differently dispersed from their means, with LG-EXP-WRI showing the most degree of dispersion.

In addition, the difference between the calculated mean statistics of two Low-Intermediate means (i.e. 17.03 and 15.93), and two High-Intermediate means (i.e. 17.60 and 17.37) shows that proficiency level has been of effect on the performance of groups; that is, there is more clustering of scores around the midpoint, and as result less variability, in the two performance types of the High-Intermediate group than the Low-Intermediate group. Hence, the more the proficiency level of a group, the more the homogeneity between the performance types of that group under different conditions (i.e. GJ tasks).

The level of significance (alpha level) set as the criterion and standard for rejection and acceptance at the onset of this study was $5 \%(.05)$. Hence, the results of the two-way repeated measures ANOVA tests, the univariate $F$-tests, would be analyzed at the .05 level to indicate that a $95 \%$ probability existed that the difference between the different performance types of the groups had been due to the experimental treatment rather than to sampling error.

The two-way repeated measures ANOVA, which compares participants' performance on more than one task, is a robust parametric test. One of the assumptions underlying parametric tests is that data points are independent, but this assumption does not hold for a repeated measures design because, in repeated measures design, data for different conditions have come from the same people, hence the relatedness of data from different experimental conditions. This issue gives rise to an additional assumption according to which the relationship between pairs of groups is equal; that is, the variances of the differences between levels of the repeated measures factor are equal in a within-subjects design. This assumption is called sphericity or circularity. This assumption resembles the homogeneity of variance assumption in between-subjects ANOVA. In fact, this assumption should not be violated because its violation causes loss of power in terms of an increased probability of a Type II Error and not being able to compare the $F$ statistic to the normal tables of $F$. In addition, the software used cannot calculate a significance value. However, the SPSS software package includes a procedure called Mauchly's test that determines if the assumption of sphericity has been violated. First, we ran the Mauchly's test to determine whether the condition of Sphericity has been met; that is, it tests the hypothesis that the variances of the differences between conditions are equal. Table 2 shows the findings of running Mauchly's test for the first hypothesis. If Mauchly's test statistic is significant (i.e. a probability value less than .05), it will indicate the existence of significant differences between the variance of differences, and, as a result, the violation of sphericity ensues; in this case, we cannot trust the $F$-ratios.

If, on the other hand, Mauchly's test statistic is nonsignificant (i.e. a probability value more than .05), it will indicate lack of significant differences between the variance of differences and, as a result, the observance of sphericity. Since the significance values, as shown in Table 2 , are $<.05$, we concluded that there was a significant difference between the two GJ tasks. In addition, there was a significant difference between the two proficiency levels, but this test does not tell us which GJ task or proficiency level differed from the other one. To decide which of the three corrections to use (i.e. whether to use Green-House Geisser, Huynh-Feldt or Lower-Bound), we checked the epsilon value in Table 2. Epsilon is a descriptive statistic that indicates the degree to which sphericity has been violated. In cases where the epsilon value is more than 0.75 (i.e. $>0.75$ ), such as the case in Table 2, we must use the Huynh-Feldt correction, whereas in cases where the Epsilon value is less than 0.75 (i.e. $<0.75$ ), we must use the Greenhouse-Geisser correction. Since in this study the Epsilon values from Mauchly's test values are 1.000 (i.e. Table 2) and all of the three values are E $>0.75$, we used the less conservative Huynh-Feldt corrected values to adjust both within-subject and between-subject degrees of freedom as shown in Tables 3, 4, and 5.

Having used the corrections in Table 2 (i.e. under the heading of Epsilon) to adjust the degrees of freedom for the averaged tests of significance, we, as a result, used the corrected tests, as shown in Tables 3, 4, and 5(i.e. tests of within-subjects effects). Applying the Huynh-Feldt correction epsilon value, as shown in Table 3, we obtained no significant differences between the two proficiency groups in terms of their performance on the two GJ tasks. That is, the corrected $F$-ratio of Huynh-Feldt Correction procedure shows the significance value of (.208) according to 
which High-Intermediate and Low-Intermediate proficiency groups showed no significant differences in their performance in the two GJ tasks over two weeks while rendering WH-questions. Hence, we cannot obtain an understanding as to which proficiency group showed significant effects in its performance in the two GJ tasks while rendering WH-questions. Applying the Huynh-Feldt correction epsilon value, as shown in Table 4, we obtained no significant differences in the effects of different treatments Grammaticality Judgement tasks (i.e. Explicit and Implicit Written GJ tasks) on the performance of female EFL participants while rendering WH-questions. That is, the corrected $F$-ratio of Huynh-Feldt correction procedure shows the significance value of (.118) according to which no significant differences existed between the two Grammaticality Judgement tasks in terms of their effects on the female EFL participants' performance while rendering WH-questions in Explicit and Implicit Written GJ tasks. Hence, we cannot obtain an understanding as to which of the two Grammaticality Judgement tasks is of the significant effect on the performance of female EFL participants while rendering WH-questions. The exact significance levels in Table 4 are the result of comparing the $F$-ratios against a critical value for 1 and 29 degrees of freedom.

Since the statistical analysis used is the two-way repeated measures ANOVA, where one factor is varied between subjects (i.e. proficiency level), and the other within subjects (i.e. Explicit and Implicit Written GJ tasks), we analyze the interaction between a within-subjects factor (i.e. the two treatments of the explicit and implicit GJ tasks) and a between-subject factor (i.e. group in terms of the two proficiency levels [High- and Low- Intermediate levels]) for the two experimental groups, but since there was violation of sphericity, we, using the Table 5, cannot look at the interaction without a correction, hence the application of the Huynh-Feldt correction epsilon value, as shown in Table 5. Applying the Huynh-Feldt correction epsilon value, as shown in Table 5, we obtained no significant interaction between the within-subjects factor (i.e. the two treatments of the Explicit and Implicit Written GJ tasks) and the between-subject factor (i.e. group in terms of the two proficiency levels) for the two experimental groups. That is, the corrected $F$-ratio of Huynh-Feldt correction procedure shows the significance value of (.361) according to which no significant interaction existed between the within-subjects factor (i.e. the two treatments of the Explicit and Implicit Written GJ tasks) and the between-subject factor (i.e. group in terms of the two proficiency levels) for the two experimental groups.

The nonsignificant interaction indicates that the groups are not changing in terms of performance over different treatment times. Hence, we cannot obtain an understanding as to whether the interaction of group and treatment is of any significant effects on the ability of female EFL participants while rendering WH-questions in either of the proficiency groups. The exact significance levels in Table 5 are the result of comparing the $F$-ratios against a critical value for 1 and 29 degrees of freedom.

Hence, Mauchly's test indicated that the assumption of sphericity had been violated (chi-square $=.000, \mathrm{p}<.05$ ), therefore degrees of freedom were corrected using Huynh-Feldt estimates of sphericity (epsilon $=1.000$ ). The results show that the performance scores of the two proficiency groups (i.e. High-Intermediate and Low-Intermediate Proficiency Groups) in the two Grammaticality Judgement tasks (Explicit Written and Implicit Written Grammaticality Judgement tasks) did not differ significantly, and, as a result, the first hypothesis was rejected, Group $\mathrm{F}(1.000,29.000)=1.660, \mathrm{P}>.05$; Treatment $\mathrm{F}(1.000,29.000)=2.601, \mathrm{p}>.05$; Group*Treatment $\mathrm{F}(1000,29.000)=.863, \mathrm{P}>.05$. . Post hoc tests revealed that the performance scores of the two proficiency groups in Explicit and Implicit Written Grammaticality Judgement tasks did not show significant differences. That is, neither group's performance was significantly better than the other group in the two GJ Tasks (i.e. Explicit and Implicit Written GJ tasks) ( $\mathrm{p}>.05)$.

\section{Discussion}

We expected L2 participants to be more successful when receiving explicit stimuli than implicit stimuli in recognizing correct and incorrect WH-questions in GJ tasks because researchers (e.g., Hayes \& Broadbent) have argued that implicit learning is a careless and passive process of gathering information about the simultaneous occurrence of various events and features. However, the results of this study have contradicted the assertions held by such researchers and have proved that both implicitness and explicitness are active mental processes largely because this study showed no significant differences between the performance of EFL female participants in the Explicit and Implicit Written GJ tasks.

In addition, we expected the High-Intermediate group to be more successful in recognizing WH-questions than the Low-Intermediate group because research (e.g., White, 1988) has indicated that High-Intermediate adult learners of English (e.g. French-speaking learners of English) could reject subjacency violations such as extractions from WH-islands because they had the right parameter settings, whereas Low-Intermediate learners were not as successful, but the results of this study showed no significant differences between the performance of 
High-Intermediate and Low-Intermediate EFL female participants in the Explicit and Implicit Written GJ tasks, hence the rejection of the contentions held by such researchers.

However, the results obtained from the statistical analysis of the performance data of Persian female EFL participants support Schachter's (1988) assertion that in cases where L2 learners' L1 does not resemble L2 in WH-movement, such as the case of Persian participants in this study, L2 learners' observance of subjacency could only be due to UG and not L2 learners' L1. In fact, participants of this study merely showed signs of L1 (i.e. Persian) interference while rendering those WH-Questions which were fillers and resembled Persian WH-questions; that is, in cases where L1 and L2 showed similarities, even the slightest ones, participants violated subjacency (even most of the High-Intermediate participants), whereas in cases where no such similarities existed, participants observed subjacency, hence the positive role of UG in this regard. Such findings oppose the stance adopted by such researchers as Bley-Vroman, Felix \& Loup (1988) because they have only emphasized UG availability while rendering GJ tasks on subjacency. The findings of this study have proved the simultaneous roles of both UG and L1 in rendering GJ tasks on subjacency.

Since the performance of High-Intermediate and Low-intermediate groups was not significant, findings oppose the assertions of White, Travis \& Maclachlan (1992) according to which all the High-Intermediate adult Malagasy learners of English, and only half of the Low-Intermediate group performed like a native English-speaking control group in an experimental study. In addition, results of this study have proved that UG principles remain available in adult L2 acquisition. However, such results contradict Bley-Vroman's belief that states that access to UG is only via the L1 because female EFL participants could properly observe subjacency in cases where sentence structures had no counterparts or equivalences in Persian. Moreover, the results have opposed the belief of those who claim no access to UG at all such as Clahsen and Muysken (1986).

This study does not support the assertions of Green and Hecht (1992) according to which explicit and implicit tests show different kinds of sensitivity to the participants' implicit and explicit knowledge. The reason is that the performance of participants on Explicit and Implicit tasks in this study was by no means statistically significant, a result indicating that Explicit and Implicit Written GJ tasks used in this study do not show different kinds of sensitivity to the participants' implicit and explicit knowledge of subjacency.

The results of this study, however, do support the findings of such research Bley-Vroman, Felix and Loup (1988) because such researchers have mostly concluded that subjacency is part of L2 learners' grammatical proficiency. Further, the results support the work of such researchers as Ellis who have viewed interlanguage as consisting of a number of interlocking systems. Explicitness and implicitness, which have been studied in this study, are only two of the many subcomponents underlying the basic structure of participants' grammatical proficiency.

Such findings do not support the assertions of Cleeremans and McClelland, (1991) who have claimed that implicit learning is restricted when subjects are to establish relationships between distant elements in sentences, and that only simple knowledge can be implicitly learned and not more complex knowledge. The reason for rejecting such assertions is that participants have shown signs of having implicit knowledge of subjacency, which is not simple but complicated.

However, the results of this study do support the claim posed by Bialystok (1992) who has indicated that the nature of the relationship between explicit and implicit knowledge is different in L2 from L1; that is, in L1 children first acquire implicit knowledge of their L1, and, afterwards, they develop explicit knowledge out of L1 by analyzing it; hence, implicit knowledge forms the basis of explicit knowledge, whereas in L2, it is the other way round except for the cases of naturalistic exposure where implicit L2 knowledge serves as the pillar for explicit L2 knowledge development. The numerical values of mean, as shown in Table 1, indicate that both High-Intermediate and Low-Intermediate groups did better in their Explicit Written GJ tasks and not in their implicit ones.

\section{Conclusion}

The rationale behind the obtained linguistic theories of the hypothesis in this study is to explain an aspect of the process of SLA in a measurable way (i.e. the degree to which explicit and implicit modes of input presentation affect the EFL learners' ability to observe subjacency). We could not establish an interaction between SLA theory-based explanations (e.g. SLA as a cognitive process) and the data gathered by administering the GJ tasks (Explicit and Implicit GJ tasks) on such constructs because no significant differences were seen between the explicit and implicit modes of receiving input, which are cognitive issues, in the performance of High-Intermediate and Low-Intermediate female EFL participants. In fact, the performance of Low-Intermediate group bore a close resemblance to the performance of High-Intermediate group in both Explicit Written and Implicit Written GJ tasks; a fact indicating that both groups were under the influence of the symbolic aspects of the WH-questions and not the cognitive-semantic relationship between the stimuli and WH-questions. The scores of most of the High-Intermediate 
and Low-Intermediate participants were rather high in both Explicit Written and Implicit Written GJ tasks (i.e. the performance of both of the groups is above 15 in both the Explicit Written and Implicit GJ tasks [15 out of 30 questions]; that is, the performance of the Low-Intermediate group was 22/30 in Explicit Written GJ task and 17/30 in Implicit Written GJ task, and the performance of the High-Intermediate group was 22/30 in Explicit Written GJ task and 21/30 in Implicit Written GJ task). A fact indicating that other factors, other than the explicit-implicit dichotomy, must have affected the performance of participants

Hence, the obtained findings from the hypothesis point to the conclusion that the generative view of language can be adhered to (i.e. language is a symbolic system which is independent of cognition) because the female EFL participants did not show significant differences in their performance in the Explicit Written and Implicit Written GJ tasks in this study. In other words, the explicit-implicit dichotomy of the GJ tasks failed to trigger participants' cognitive processing capabilities despite the fact that participants were in two proficiency levels (i.e. the reason is that participants primarily focused on the symbolic aspects of WH-questions and not the semantic-cognitive relations between the explicit-implicit stimuli and WH-questions). A fact that leads us to conclude that female EFL participants are primarily under the influence of the symbolic aspects of the L2. Hence, language is indeed highly complex and such learning strategies as inductive learning strategies, deductive strategies, or rather general problem-solving strategies can by no means lead to language acquisition.

The overall conclusion drawn from the results and discussion of the hypothesis is that the examination of the nature of female EFL participants' grammatical proficiency from the explicit-implicit standpoint does not result in viewing the explicit-implicit dichotomy as a distinct component part of female EFL participants' grammatical proficiency. That is, the adoption of a separate explicit-implicit approach towards the investigation of the nature of L2 grammatical proficiency does not trigger distinct levels of comprehension of English WH-questions on EFL female participants' part. Hence, this issue cannot lead to a major shift in SLA researchers' long-held view towards EFL learners' grammatical proficiency because the hypothesis attests to the long-held view of grammatical proficiency as a unified solid whole and not a unit made of numerous component parts with each part being triggered by a distinct task type; otherwise, EFL female participants would have shown significant differences in their performance in the Explicit and Implicit GJ Tasks.

Accordingly, such independent variables as the implicit way of input presentation and the explicit way of input presentation do not have different effects of the EFL female participants' comprehension of English WH-questions; that is, High-Intermediate and Low-Intermediate EFL female participants perform similarly across the explicit and I

implicit independent variables because they use the same cognitive, linguistic, and psychological basis for processing such variables. In fact, explicit and implicit means of receiving linguistic input do not have different effects on female EFL participants' language processing because similar psychological processes are triggered by explicit and implicit types of linguistic input. Hence, the explicit-implicit dichotomy does not serve as a separate subcomponent of L2 grammatical proficiency because the explicit and implicit forms of linguistic input have similar effects on female EFL learners' performance in the Explicit Written and Implicit Written GJ tasks. Teachers can adopt a single teaching method for the practical use of explicit and implicit teaching stimuli while teaching subjacency operations to EFL learners of different proficiency levels.

The findings of this study can also improve and modify the theoretical foundations of Testing towards the use of GJ tasks because GJ tasks have proved not to be of that much practical use in testing the explicit-implicit knowledge of L2 learners; hence, researchers should not use this type of test if they intend to measure the explicit and implicit knowledge of their L2 learners. Furthermore, different schools, language institutes, and universities can revise their use of GJ tasks and make use of other tasks. In fact, such academic organizations should reevaluate the success of GJ tasks in the extent to which such tasks can properly elicit the use of a target structure, such as WH-questions.

\section{References}

Collins, P. (2006). Clause types. In: B. Aarts \& A. McMahon (Eds.), The handbook of English Linguistics (pp: 180197). Blackwell Publishing.

Cook, V. (1993). Linguistics and Second Language Acquisition. The Macmillan Press Ltd.

Dekeyser, R. (2003). Implicit and explicit learning. In: C.J. Doughty \& M.H. Long (Eds.), The handbook of Second Language Acquisition (pp: 313-348). Malden: Blackwell Publishing Ltd.

Ellis, R. (2003). Task-based language learning and teaching. Oxford University Press.

Fromkin, V., Rodman, R. \& Hyams, N. (2003). An introduction to language ( $7^{\text {th }}$ ed.). MA: Thomson Heinle. 
George, A. (1989). How not to become confused about linguistics. In: A. George (Ed.), Reflections on Chomsky (pp: 90-110). Oxford: Blackwell Publishers.

Haegeman, L. (1991). Introduction to Government and Binding Theory. Cambridge: Cambridge University Press. Hawkins, R. (2001). Second language syntax: A generative introduction. Oxford: Blackwell Publishing.

Huddleston, R. \& Pullum, G. K. (2006). Coordination and Subordination. In: B. Aarts \& A. McMahon (Eds.), The handbook of English Linguistics (pp: 198-219). Blackwell Publishing.

Isac, D.,\& Reiss, C. (2008). I-Language: An introduction to Linguistics as Cognitive Science. Oxford University Press.

Lotfi, A.R. (2003). Intonation contours, yes/no questions, and minimalist syntax: A unitarianist perspective. Cognitive Sciences, 70-83. Esfahan: Azad University at Esfahan.

Matthews, P.H. (2007). Oxford concise dictionary of linguistics $\left(2^{\text {nd }}\right.$ ed.). Oxford: Oxford University Press.

Radford, A. (1997). Syntax. A minimalist introduction. Essex: Cambridge University Press.

Radford, A. (2004). Minimalist syntax: Explaining the structure of English. Cambridge: Cambridge University Press.

Radford, A., Atkinson, M., Britain, D., Clahsen, H. \& Spencer, A. (2009). Linguistics: An introduction (2 ${ }^{\text {nd }}$ ed.). Cambridge: Cambridge University Press.

Ramchand, G. C. (2008). Verb meaning and the lexicon: A first-phase syntax. Cambridge University Press.

Scalise, S., Magni, E. \& Bisetto, A. (Eds.) (2009). Universals of language today: Studies in natural language and linguistic theory. Springer.

Skehan, P. (2008). Interlanguage and language transfer. In: B. Spolsky \& F.M. Hult (Eds.), The handbook of educational linguistics (pp: 411-423). MA: Blackwell Publishing Ltd.

White, L. (1988). Island effects in Second Language Acquisition. In: S. Flynn \& W. O'Neil (Eds.), Linguistic theory in Second Language Acquisition (pp: 144-172). Dordrecht: Kluwer Academic Publishers.

White, L. (1989). Universal Grammar and Second Language Acquisition. Philadelphia: John Benjamins Publishing Company.

White, L. (1996). Universal Grammar and Second Language Acquisition: Current trends and new directions. In: n.d. (eds.), Handbook of Second Language Acquisition (pp: 85-120). Academic Press, Inc.

White, L. (1990). Second Language Acquisition and Universal Grammar. SSLA (PP: 121-133). USA: Cambridge University Press.

Table 1. Sample Descriptive Statistics

\begin{tabular}{|l|r|r|r|r|r|r|}
\hline $\begin{array}{l}\text { Independent } \\
\text { Variables }\end{array}$ & $\mathrm{N}$ & \multicolumn{1}{c|}{ Range } & Mean & Standard Error of the Mean & Standard Deviation & Variance \\
\hline LG-EXP-WRI & 30 & 15 & 17.03 & .726 & 3.978 & 15.826 \\
\hline LG-IMP-WRI & 30 & 11 & 15.93 & .538 & 2.947 & 8.685 \\
\hline HG-EXP-WRI & 30 & 11 & 17.60 & .513 & 2.811 & 7.903 \\
\hline HG-IMP-WRI & 30 & 13 & 17.37 & .568 & 3.113 & 9.689 \\
\hline
\end{tabular}

Table 2. Mauchly's Test of Sphericity

\begin{tabular}{|c|c|c|c|c|c|c|c|}
\hline \multirow[b]{2}{*}{$\begin{array}{l}\text { Within-Subjects } \\
\text { Effect }\end{array}$} & \multirow[b]{2}{*}{ Mauchly’s W } & \multirow[b]{2}{*}{$\begin{array}{r}\text { Approx. Chi } \\
\text { Square }\end{array}$} & \multirow[b]{2}{*}{$\mathrm{df}$} & \multirow[b]{2}{*}{ Sig. } & \multicolumn{3}{|c|}{ Epsilon............... } \\
\hline & & & & & $\begin{array}{l}\text { Greenhouse- } \\
\text { Geisser }\end{array}$ & $\begin{array}{l}\text { h- Lor } \\
\text { Feldt }\end{array}$ & Bound \\
\hline Group & 1.000 & .000 & 0 & . & 1.000 & 1.000 & 1.000 \\
\hline Treatment & 1.000 & .000 & 0 & . & 1.000 & 1.000 & 1.000 \\
\hline Group*Treatment & 1.000 & .000 & 0 & . & 1.000 & 1.000 & 1.000 \\
\hline
\end{tabular}


Table 3. Tests of Within-Subjects Effects

\begin{tabular}{|l|c|r|r|r|c|}
\hline Source & Type III Sum of Squares & df & Mean Square & F & Sig. \\
\hline Group Sphericity Assumed & 30.000 & 1 & 30.000 & 1.660 & .208 \\
\hline Greenhouse-Geisser & 30.000 & 1.000 & 30.000 & 1.660 & .208 \\
\hline Hynh-Feldt & 30.000 & 1.000 & 30.000 & 1.660 & .208 \\
\hline Lower-Bound & 30.000 & 1.000 & 30.000 & 1.660 & .208 \\
\hline
\end{tabular}

Table 4. Tests of Within-Subjects Effects

\begin{tabular}{|l|l|l|l|l|c|}
\hline Source & Type III Sum of Squares & df & Mean Square & F & Sig. \\
\hline Group Sphericity Assumed & 13.333 & 1 & 13.333 & 2.601 & .118 \\
\hline Greenhouse-Geisser & 13.333 & 1.000 & 13.333 & 2.601 & .118 \\
\hline Hynh-Feldt & 13.333 & 1.000 & 13.333 & 2.601 & .118 \\
\hline Lower-Bound & 13.333 & 1.000 & 13.333 & 2.601 & .118 \\
\hline
\end{tabular}

Table 5. Tests of Within-Subjects Effects

\begin{tabular}{|l|l|l|r|r|r|}
\hline Source & Type III Sum of Squares & \multicolumn{1}{c|}{ df } & Mean Square & F & Sig. \\
\hline Group* Sphericity Assumed Treatment & 5.633 & 1 & 5.633 & .863 & .361 \\
\hline Greenhouse-Geisser & 5.633 & 1.000 & 5.633 & .863 & .361 \\
\hline Huynh-Feldt & 5.633 & 1.000 & 5.633 & .863 & .361 \\
\hline Lower-Bound & 5.633 & 1.000 & 5.633 & .863 & .361 \\
\hline
\end{tabular}

